\title{
Coffee sludge as a new food ingredient
}

\author{
Bagrat Khashpakyants, Irina Krasina*, and Elizaveta Filippova \\ Kuban State Technological University, 2, Moskovskaya st, Krasnodar, 350072, Russia
}

\begin{abstract}
Spent coffee grounds (coffee sludge) is the most common recycled material obtained in the production of instant coffee. Thus, about 6 million tons of coffee sludge are generated annually. The purpose of this study was to evaluate the use of coffee sludge as an innovative functional food ingredient in flour confectionery products and to study the effect of coffee sludge and its chemical components on sensory properties, volatile components and rheological properties of the final product. Spent coffee grounds contain significant amounts of insoluble dietary fiber, proteins, lipids, ash and lower glycemic sugars. Mixing wheat flour with coffee sludge did not affect significantly the rheological properties of the dough and overall acceptability of the final product. Thus, the obtained biscuits can be recommended for patients with diseases associated with obesity and diabetes, as well as for people who prefer low-calorie foods.
\end{abstract}

Coffee is one of the most popular beverages in the world, with over 400 billion cups served annually. Coffee is grown in more than 50 developing countries in South and Central America, Africa, Asia and the Caribbean. ICO 2020 Annual Production Report showed 153.9 million sacks ( $60 \mathrm{~kg}$ each), of which 97, 3 million sacks of Arabica and 56.6 million sacks of Robusta. [1]. Simply stated, coffee is a beverage made by extracting the soluble material of roasted coffee grounds using boiling water. Various methods of coffee preparation are used in the world, depending on the taste and requirements of consumers, for example, the percolation filtration method. The coffee industry, as one of the most important agroindustrial and food sectors, produces large quantities of waste, both liquid and solid. Spent coffee grounds (coffee sludge) is the most common residue, which makes up $45 \%$ during the processing of coffee beans, obtained during the treatment of coffee beans with hot water or steam for the instant coffee preparation. Thus, it generates about 6 million tons of coffee sludge annually, as $50 \%$ of the world's coffee production is used to make instant coffee.

Nutritionally, coffee sludge contains significant amounts of dietary fiber $(47.3 \mathrm{~g} / 100 \mathrm{~g}$ coffee sludge), composed mainly of insoluble fiber (88\%). [2-4]. Polysaccharides constitute the other carbohydrate resource found in coffee sludge (13.1\%), while free glucose is found in negligible concentrations $(0.04 \mathrm{~g} / 100 \mathrm{~g}$ coffee sludge), which means the lower glycemic index of sugars. The ash content of coffee sludge is $1,30 \%$, including many elements, e.g. potassium, sulfur, calcium, iron, magnesium, phosphorus, copper, manganese, boron and others. Unsaturated fatty acids are the main lipids in coffee sludge, which constitute from 11 to $20 \%$ of the total lipidic weight). At the same time, the protein content ranges from 6.7-9.9

\footnotetext{
* Corresponding author: kib6060@mail.ru
} 
to $12.8-16.9 \%$ due to the different conditions used in the manufacture of the instant coffees [5-8].

Despite promising nutritional properties of coffee sludge, it is used in industrial fields as a fuel in boilers due to its high calorific power [9,10], as feed for pigs, ruminant animals, chickens and rabbits [11-13] and as the potential source for fuel pellets and biodiesel production $[14,15]$.

As far as modern lifestyle is concerned, consumers are looking for healthy foods with a lower glycemic index as well as low calorie foods. These are the main problems to be solved in the food industry today, in addition to the problem of dietary fiber content in foods and their role in a balanced diet [16-18]. Health officials strongly recommend low-glycemic foods which can help resolve the problem of obesity and related diseases such as diabetes. The purpose of our study was to assess the possibility of using coffee sludge as an innovative functional food ingredient in flour confectionery products and to study the effect of coffee sludge and its chemical components on sensory properties, volatile components and rheological properties of the final product.

During the investigation of the chemical composition of the coffee sludge it was found out that the moisture content was $58.98 \%$, therefore, it was necessary to dry the coffee sludge in order to control the microbiological activity, which can contribute to the decomposition of the product and can also interfere with fat extraction. After vacuum drying, the moisture content of the coffee sludge was $7.47 \%$ (Table 1), which corresponds to the technological and quality recommendations, which provide for a moisture content below $10 \%$.

Table 1. Chemical composition of raw materials and finished biscuits

\begin{tabular}{|c|c|c|c|c|c|c|}
\hline \multirow[t]{3}{*}{ Index, $\%$} & \multicolumn{2}{|c|}{ raw materials } & \multicolumn{4}{|l|}{ biscuits } \\
\hline & \multirow[t]{2}{*}{ Wheat flour } & \multirow{2}{*}{$\begin{array}{l}\text { Coffee } \\
\text { sludge }\end{array}$} & \multirow{2}{*}{$\begin{array}{l}\text { Control } \\
\text { sample }\end{array}$} & \multicolumn{3}{|c|}{ dosage of coffee sludge, $\%$} \\
\hline & & & & 2 & 4 & 6 \\
\hline Moisture & 11.90 & 7.47 & 5.76 & 6.30 & 7.02 & 7.88 \\
\hline Protein & 12.05 & 8.97 & 8.23 & 7.32 & 6.05 & 6.78 \\
\hline Fat & 1.12 & 13.89 & 16.10 & 16.15 & 16.17 & 16.37 \\
\hline $\begin{array}{l}\text { Carbohydrates, } \\
\text { including }\end{array}$ & 74.23 & 63.90 & 73.71 & 74.26 & 75.00 & 73.98 \\
\hline Dietary fiber & 0.45 & 51.86 & 1.06 & 1.10 & 1.13 & 1.16 \\
\hline Ash & 0.70 & 2.77 & 0.90 & 1.22 & 1.65 & 1.71 \\
\hline
\end{tabular}

Due to its hydrophilic nature of the inorganic minerals and the different processing conditions used in the preparation of instant coffee, the ash content decreased from 4.6 to $2.77 \%$.

The coffee sludge contains a significant amount of fat (13.8 9\%), as shown in Table 1, since beans of Robusta and Arabica have a high percentage of lipids - 11-16\% and 14-20\%, respectively, but, they are almost not extracted with hot water during the production of instant coffee and, therefore, remain in the coffee sludge. Lipids contribute to the good sensory profile of coffee, and contain fatty acids such as linoleic acid (40\%), palmitic acid (30\%), oleic acid (10\%) and stearic acid (7\%), which are the main components of these lipids.

Dietary fiber is the main component among others in coffee sludge $(51.86 \%)$. Insoluble fiber is the main constituent of dietary fiber $(88 \%)$, which is predictable in terms of the process and recovery of most of the soluble fiber during the production of instant coffee. Dietary fibers of coffee sludge are rich in lignocellulose, a covalently bonded network of polysaccharides of lignin, cellulose and hemicellulose, which gives structural stability to the plant cell wall. Dietary fibers are not digested in the small intestine, but fully or partially digested, fermented in the large intestine and, therefore, organize its functions and have important physiological effects on glucose, lipid metabolism and mineral bioavailability. 
Nowadays, dietary fibers are known to be protective effect against certain gastrointestinal diseases, such as colon cancer, duodenal ulcers, cardiovascular disease, stroke, diabetes, constipation, hemorrhoids, gastroesophageal reflux disease, diverticulitis, obesity and hypertension. Dietary fibres also have technological and functional properties which can be used in the formulation of foods, as well as numerous beneficial effects on human health.

The chemical composition of the control and experimental samples of cookies is presented in Table 1. Coffee sludge contains a certain amount of fiber, as well as ash, which is a source of potassium, magnesium, calcium, sulfur and phosphorus. When the dosage coffee sludge increased from 2 to $6 \%$, in response, fibre and ash content of the experimental biscuits also increased compared to the control sample. (Table 1). The protein content of samples supplemented with coffee sludge was lower compared to the control sample, while the fat content did not show a significant difference $(\mathrm{p}>0.05)$.

Most active compounds formed during baking are products of non-enzymatic browning or Maillard reactions; therefore, the extent of browning reactions was investigated using both instrumental and sensory methods, but from different angles. The tasters were asked not to give their opinion on the tone color, but the instrumentation data were expressed as tricolor parameters associated with the tone color of cookies.

Wheat flour had higher whiteness index $\left(\mathrm{L}^{*}=92.33\right)$, while the coffee sludge was dark $(\mathrm{L} *=20.05)$. Produced biscuit indicated, that whiteness $\left(\mathrm{L}^{*}\right)$ of control sample reached to 71.17 , while mixing coffee sludge with wheat flour at levels 2,4 and $6 \%$ decreased $\mathrm{L}^{*}$ to 66.31, 54.88 and 43.88, respectively. The decreasing level of whiteness $\left(\mathrm{L}^{*}\right)$ with increased concentration of dark coffee sludge is explained by the phenomenon of scattering of light. Also, redness degree $\left(a^{*}\right)$ was lower in wheat flour $(0,45)$ than in coffee sludge $(3,57)$. Consequently, a* value of biscuit increased with increasing mixing level of coffee sludge, where $a^{*}$ value was 2.53 in control sample, then increased significantly at mixing level 2, 4 and $6 \%$ to $2.92,3.41$ and 5.62. In contrast to the $a^{*}$ value, the yellowness $b^{*}$ value was higher in wheat flour (9.53) than in coffee sludge (6.58), therefore, mixing wheat flour with coffee sludge at 2, 4 and $6 \%$ decreased significantly $b^{*}$ value in biscuit from 24,52 in control sample to $21.17,18.12$ and 15.49 . It is well known that, the increase of $a^{*}$ and $b^{*}$ corresponds to the increase in redness and yellowness, respectively, which occurs in the beginning of nonenzymatic browning or Maillard reaction. However, an increase in $\mathrm{a}^{*}$ values and a decrease in $b^{*}$ might be due to the fact that the brown color is too dark to be detected. Therefore, such values cannot be taken as indicators for the development of browning reaction during our study. It was established that in the control cookie sample, a higher formation of Amadori compounds occurs to a greater extent than in the experimental samples with coffee sludge and other ingredients, which indicates a faster rate of the Maillard reaction at an early stage. This can be explained by the lower content of glycemic sugars, which can be found in the test samples, which affected negatively on browning development.

Thirty-eight volatiles were isolated and identified in cookie samples by using SPME/GCMS analysis. These compounds belong to different chemical classes, mainly aliphatic aldehydes (12), short-chain and fatty acids (6), furans and furanones (7), and pyrazines (4). These volatile substances were formed by the Maillard reaction, lipid oxidation, or fermentation.

The 2/3- Methylbutanal and Phenylacetaldehyde, which were identified in our study, are known as Strecker's aldehydes, derived from isoleucine, leucine, and phenylalanine, respectively. An increase in the dosage of coffee sludge revealed a slight decrease in the Strecker aldehydes concentrations, due to a lower content of reducing sugars in it, which affected negatively the development of the early stage of Maillard reaction, leading to the formation of such aldehydes.

Lower glycemic sugars can be easily decomposed during non-enzymatic browning, resulting in the formation of many compounds, such as 2,3-Butanedione and 2,3- 
Pentanedione. However, the decrease in the amounts of such diketones upon supplemented biscuits with coffee sludge, revealed the lower glycemic index of these residues.

Furans were the main class among the volatiles of both the control sample and supplemented samples, while 2-Furfural constituted the predominant compound, but decreased significantly ( $\mathrm{p}<0.05$ ), when the level of coffee sludge increased. Furfural is pungent and sweet, but lacks the cookie-like caramel flavor. However, 2-Ethyl-5methylfuran and 2-Pentylfuran are lipid derived products with a non-significant increase ( $\mathrm{p}>$ $0.05)$ on increasing the dosage of coffee sludge. 2,5-Dimethyl-4-hydroxy-3 (2H) -furanone is the only furanone identified with caramel, sweet, fruity, nutty and burnt notes.

Pyrazines are characterized by their roasted nutty flavor. The reaction of amino acids with sugar is the main pathway for the formation of alkyl pyrazines, but the inhibition or the decrease in the rate of browning due to lack of reducing sugars, may decrease pyrazines with increasing coffee sludge.

As 2,3-Dihydro-3,5-dihydroxy-6-methyl-4(H)-pyran-4-one and its thermal dehydrated product, 3-hydroxy-2-methyl-4 (H) -pyran-4-one (maltol) have sweet-caramel note and wellknown pyranones in cookies. Addition of protein source to cookie dough promotes the generation of pyranones with a pleasant sweet aroma. Thus, biscuits samples supplemented with coffee sludge showed lower content of pyranones in comparison to the control sample due to the lower protein content of the supplemented recipes.

Oxidation of oleic acid, linoleic acid and linolenic acid, which are found in wheat flour and coffee sludge, is responsible for the formation of products of lipid degradation in cookies, such as hexanal, nonanal, heptenal and 2,4-decadienal. The apparent increase in lipid-derived volatiles upon supplemented with coffee sludge may be attributed to the decrease in the formation of Maillard- derivatives.

The mean sensory scores of the control and experimental biscuits on a 20-point scale are shown in Table 2. Significant differences $(p<0.05)$ in color were observed between the control and samples supplemented with coffee sludge, while increasing the dosage of coffee sludge significantly decreased the color score due to their dark nature.

Table 2. Effect of coffee sludge on the sensory properties of biscuits

\begin{tabular}{|l|c|c|c|c|}
\hline \multirow{2}{*}{ Index } & \multirow{2}{*}{ Control salmpe } & \multicolumn{3}{|c|}{ Dosage of coffee sludge } \\
\cline { 3 - 5 } & & 2 & 4 & 6 \\
\hline Colour & 18.15 & 16.35 & 15.01 & 13.20 \\
\hline Taste & 18.00 & 18.25 & 18.65 & 16.02 \\
\hline Aroma & 18.21 & 18.05 & 17.82 & 16.74 \\
\hline Texture & 18.25 & 18.10 & 18.44 & 18.70 \\
\hline Appearance & 18.72 & 18.47 & 17.20 & 16.14 \\
\hline
\end{tabular}

Despite the established differences between the samples in the detected volatiles, which influenced the aroma and taste, however, there were no significant differences ( $>>0.05)$ between the control samples and samples supplemented with additives ( 2 and $4 \%$ coffee sludge).

While mixing biscuits with $6 \%$ coffee sludge, aroma and taste decreased significantly $(\mathrm{p}<0.05)$. Obviously, the presence of more glycemic reduced sugar influenced the acceptability of smell and taste, which is the reason for their responsibility for the development of the browning reaction and generation of compounds with sensory characteristics, for example, roasted, sweet and caramel.

The texture of the supplemented samples with additives seems to be nearly the same as the control sample or exceeds it, without significant differences $(p>0.05)$, which may be attribute to the higher emulsifying activity and stability of the coffee sludge. Low appearance scores for the sample supplemented with $6 \%$ coffee sludge, may be due to darkening of the 
product. However, overall acceptability did not show significant differences $(p>0.05)$ among the samples studied, which makes coffee sludge a promising functional ingredient with great potential to be used in flour confectionery.

The introduction of coffee sludge in the production of biscuits influenced its quality and texture characteristics. Biscuit height increases significantly with increasing mixing level of coffee sludge $(p<0.05)$, while the volume showed a significant rise upon addition of coffee sludge generally. This effect may be due to the high fiber content of the coffee sludge, as well as to the emulsifying properties. Meanwhile, diameter and spread ratio were significantly deceased in the test samples compared to the control sample, in which coffee sludge was added.

A texture profile curve (strength depending on time) was conducted to determine the hardness and resilience of the cookie samples. Hardness is the peak force measured during the first compression cycle. Resilience is how well a product "fights to regain its original position." Measurement of the biscuit texture in a texture analyzer showed that the hardness value decreased when the dosage of coffee sludge was increased in the biscuit formulation. The harder texture of the cookie is attributed to the increased protein content and protein interactions during dough development and baking. Variation in texture profile analysis due to various levels of coffee sludge may be due to lipid content as well as protein and starch quality. Fat coats the surface of the flour particles, inhibiting the development of a gluten framework. Therefore, the free fat disrupts the gluten network, resulting in softer doughs.

\section{References}

1. ICO., 2020. International Coffee Organization, London https://www.ico.org/

2. B. O. Khashpakyants, I.B.Krasina, P.S. Krasin. University Proceedings. Food technology, 4, 79-80 (2015) https://ivpt.kubstu.ru/tocs/346/25

3. F. Acevedo, M. Rubilar, E. Scheuermann, B. Cancino, E. Uquiche, M. Garces, J. Biobas. Mat. Bioen., 7(3), 420-428 (2013) https://doi.org/10.1166/jbmb.2013.1369

4. J. Brendan, H. Tien. Resour. Conserv. Recycl., 128, 110-117 (2017) https://doi.org/10.1016/j.resconrec.2017.10.001 .

5. B. O. Khashpakyants, I. B. Krasina, Scientific works of KubSTU. 14, 334-339 (2016) https://ntk.kubstu.ru/data/mc/0035/1453.pdf

6. É. M. D. Santos, L .M. D. Macedo, L. L. Tundisi, M. B. P. P .Oliveira, P. G. Mazzola, Trends Food Sci. Technol., 111, 280-291 (2021) https://doi.org/10.1016/j.tifs.2021.02.064

7. J. Marto, L.F. Gouveia, B.G. Chiari, A. Paiva, V. Isaac, P .Pinto, P. Simõesc, A.J. Almeidaa, H.M. Ribeiro, Ind. Crops Prod., 80, 93-100 (2016) https://doi.org/10.1016/j.indcrop.2015.11.033

8. C. Carriço, H. M. Ribeiro, J. Marto, Ind. Crops Prod., 125, 72-84 (2018) https://doi.org/10.1016/j.indcrop.2018.08.092

9. P. Döhlert, M. Weidauer, S. Enthaler, J Energy Chem., 25(1), 146-152 (2016) https://doi.org/10.1016/j.jechem.2015.11.012

10. C.M. Galanakis, Handbook of Coffee Processing By-Products. Sustainable Applications (Academic Press, 2017)

11. N. Caetano, V. Silva, A. Melo, A. Martins, T. Mata, Clean Techn. Envir. Policy, 16, 14231430 (2014) https://doi.org/10.1007/s10098-014-0773-0.

12. L. Blinová, M. Sirotiak, A. Bartosová, M. Soldán, Res. Pap. Faculty Mat. Science Tech. Trnava, 25, 91-101 (2017) https://doi.org/10.1515/rput-2017-0011

13. R. Campos-Vega, G. Loarca-Piña, H .A. Vergara-Castañeda, B. D. Oomah, Trends Food Sci. Technol., 45, 24-36 (2015) https://doi.org/10.1016/j.tifs.2015.04.012 
14. I. S. Choi, S. G. Wi, S.-B. Kim, H.-J. Bae, Biores. Technol., 125, 132-137 (2012) https://doi.org/10.1016/j.biortech.2012.08.080

$\begin{array}{llllll}\text { 15. S. K. Karmee, } & \text { Waste } & \text { Manag., } & \text { 72, 240-254 } & \text { (2018) }\end{array}$ https://doi.org/10.1016/j.wasman.2017.10.042

16.I. B. Krasina, B. O. Khashpakyants, E. V Filippova, E. V. Krasina, News of Institutes of Higher Education. Food technology, 2-3 (374-375), 42-45 (2020) https://doi.org/10.26297/0579-3009.2020.2-3.11

17.V. A. Bonilla-Hermosa, W. F. Duarte, R. F. Schwan, Biores. Techn., 166, 142-150 (2014) https://doi.org/10.1016/j.biortech.2014.05.031

18. A. Iriondo-DeHond, N. Aparicio García, B. Fernandez-Gomez, E. Guisantes-Batan, F. Velázquez Escobar, G. P. Blanch, M. I. San Andrese, S. Sanchez-Fortune, M.D. del Castillo, Innov. Food Sc. Emer. Technol., 51, 194-204 (2019) https://doi.org/10.1016/j.ifset.2018.06.010 